\title{
Support of medication adherence by community pharmacists in Czech and Slovak Republics: a questionnaire survey study
}

\author{
${ }^{1}$ Slovak Medical University in Bratislava, \\ Faculty of Public Health, Bratislava, Slovak Republic \\ ${ }^{2}$ Charles University, Faculty of Pharmacy in \\ Hradec Králové, Department of Social and \\ Clinical Pharmacy, Hradec Králové, Czech Republic \\ ${ }^{3}$ Comenius University in Bratislava, Faculty of Pharmacy, \\ Department of Organisation and Management \\ of Pharmacy, Bratislava, Slovak Republic \\ ${ }^{4}$ Comenius University in Bratislava, \\ Faculty of Mathematics, Physics and Informatics, \\ Department of Nuclear Physics and Biophysics, \\ Bratislava, Slovak Republic
}

Received 28 November, 2016, accepted 24 March, 2017

Abstract Introduction: Intervention of pharmacists in medication adherence can meaningfully contribute to achieving therapeutic outcomes. Exploring the real-life readiness and opportunities of pharmacists may result in the adoption of measures, which could be seen through improvement of patients' adherence to pharmacotherapy.

Aim: The aim of the paper was to make a survey on community pharmacists' potential in medication adherence support in its connectivity to technical and personnel factors, which underline the capacities of pharmacies in dealing with medication adherence.

Methods: The questionnaire survey was conducted from October to December 2014 and involved 158 pharmacists from 117 Czech (CZ) and 41 Slovak (SK) community pharmacies. The structured questionnaire surveyed both technical and personnel factors, including provision of consultancy services related to medication adherence. Non-adherence risk reduction was evaluated by adopting Morisky Scale modified from the pharmacist's perspective. Questionnaires outcomes were summarised in contingency tables and analyzed for associations between respective categorical variables using $x^{2}$ or exact tests and association coefficients. All results are reported as significant at $\mathrm{P} \leq 0.05$.

Results: The average score of adherence support (CZ/SK 1.95/1.93) was significantly higher as compared to that of persistence or concordance $(P<0.001)$. Reduction of non-adherence risk reached the score of a medium degree $(P=0.73$, average 2.29 in $C Z$ and 2.22 in SK). These findings were significantly associated with personnel capacities (provision of consultancy, preference for the use of recommended procedures in CZ ( $P<0.001)$, number of years of practice in $S K(P=0.029)$ ), while significant association with technical equipment (consultancy room) in the SK ( $\mathrm{P}=0.037)$.

Conclusion: The pharmaceutical care is developing towards the improvement of medication adherence in both countries - assuming a medium degree of adherence support. Further progress may be observed in strengthening the pharmacists' personnel capacities, and accelerated mainly using information technologies, i.e. through technical capacities.

Keywords community pharmacy-consultancy-medication adherence-Czech and Slovak Republics

\section{INTRODUCTION}

Modern public healthcare accentuates the importance of professional capacities of pharmacists from the perspective of their engagement in integrated and evidence-based pharmaceutical practice. Such practice is expected to expand towards and consolidate existing expertise in the areas of responsible use of medicines, innovation, disease prevention and treatment. This will also involve cooperation with other healthcare practitioners, and above all, with patients (FIP, 2011).

With the onset of the development of modern pharmaceutical care, the findings garnered within the field of clinical pharmacy have been progressively applied to the practice of community pharmacies. This means that, along with hospital and clinical pharmacists, the pharmacists practicing in community pharmacies are also continuously becoming more engaged in dealing with drug related problems (DRPs) (PCNE, 2006). The most common problems include drug interactions, adverse effects and dosage regimes, but the pharmacists' attention is also continuously shifting towards issues of medication adherence. Adherence of patients to treatment is a fundamental requirement for achieving therapeutic effect. Not complying with a prescribed therapeutic regime leads

*E-mail: milica.molitorisova@gmail.com

(c) European Pharmaceutical Journal 
to therapeutic failure and increased costs for the treatment of diseases (Kriška et al., 2015, Spinewine et al., 2012). It is a well-known worldwide problem that not only affects acute illnesses, but more frequently chronic diseases. According to the WHO (2003), up to $50 \%$ of chronically ill patients do not comply with the recommended treatment regime. Therefore, innovative and more effective multidisciplinary approaches are being sought that would improve patients' adherence, built upon an equal relationship with patients and the systematic improvement of patients' health literacy (Spinewine et al., Taitel et al., 2012). A promising approach is to link adherence with pharmacovigilance, which has become an indispensable part of the responsible use of medicines (Leporini, 2014, Sun et al., 2014). In practice, interventions are used to improve medication adherence; recently, with the help of wireless and mobile technologies, for instance, to improve dosage regimes (Středa \& Hána, 2016). Essentially, there is an increasing array of evidence that non-adherence can be effectively tackled by two complementary approaches: firstly, via a multidisciplinary approach, and secondly, via electronic and computerized healthcare systems (e-Health). If pharmacists are to be a part of this, monitoring and systemic support of medication adherence should become one of the priorities of their practice and of drug dispensation related activities.

Despite continuous scientific evaluation of pharmacists' involvement in DRPs, medication adherence including, treatment satisfaction and patient education programs, only a limited number of studies have been performed concerning the role of community pharmacists in DRPs in the Czech (CZ) and Slovak (SK) Republics (Masaryková et al., 2014, Vlček et al., 2009). A review of the existing literature reveals that there has been no research whatsoever on medication adherence support or on the reduction of non-adherence risks by community pharmacists (this is also the case specifically with regards to the technical and personnel capacities of community pharmacies). Therefore, further investigations conducted in this area could rectify this lacuna. This work has a character of a pilot study. It might contribute to the evaluation of hypothesis on a non-adherence risk reduction by pharmacists and detection in which form and with what intensity medication adherence could be supported by community pharmacists in CZ and SK. In addition, we have also focused on counselling and consultations provided by pharmacists in order to determine personnel and technical factors of pharmacies that may correlate with support for adherence in both countries.

\section{METHODS}

\section{Study design and participants}

Questionnaire survey on adherence support was provided by 158 community pharmacists from CZ and SK community pharmacies in a period from November till December of 2014. The CZ data set was composed of 117 community pharmacists (one pharmacist from one community pharmacy), that represented $56.5 \%$ of 207 pharmacies grouped in the association of independent pharmacists and in cooperation with the Faculty of Pharmacy in Hradec Králové, Charles University. From a geographical perspective, all 14 regions of $\mathrm{CZ}$ were covered.

The SK data set was composed of 41 community pharmacists (one pharmacist representing one community pharmacy) out of $128(31.8 \%)$ that we approached via students of the Faculty of Pharmacy, Comenius University in Bratislava) undertaking their mandatory practice there. From a geographical perspective, 7 out of 8 regions of SK were covered.

We are conscious of the fact that there is no specification of the Good Pharmacy Practice, which would be available exclusively for the selected data sets of CZ and SK pharmacists-responders, as compared to other CZ and SK community pharmacists, and could therefore represent a systematic error in this regard.

We used a structured questionnaire in the Czech and Slovak languages to estimate the pharmacists' support for medication adherence. The questionnaire was addressed to the heads of pharmacies or to their deputies, who answered about their practice in a specific pharmacy. It contained 15 questions divided into 5 categories that enabled us to map the basic characteristics of pharmacies, such as a geographic distribution, size, as well as specific technical and personnel factors. The aim was to examine the intensity and extent of pharmaceutical patient-oriented care from the perspective of the pharmacists taking part in this survey, regarding the surveyed medication adherence promotion provided in a pharmacy. The questionnaire was available online (Fig. 1).

\section{Structure of the questionnaire}

I. Geographic distribution of pharmacies in 5 categories according to the number of inhabitants in the local region: up to 4,999 , from 5,000 to 9,999 , from 10,000 to 49,999 , from 50,000 to 99,999 and above 100,000 inhabitants;

II. Technical equipment of pharmacies, meaning the spatial, technical and technological capacities of pharmacies, which are determined according to the legal requirements on pharmaceutical care provision in CZ (Decree No 92/2012) and SK (Decree No 129/2012). In the questionnaire, we have focused on spatial capacities-space for confidential conversation with the patient designated for counselling and consultations;

III. Personnel capacities of community pharmacies, meaning the number of professional employees, and the number of years of professional experience of the respondent and his or her qualification; engagement of pharmacists into counselling and consultancy;

IV. Counselling and consultations provided by pharmacists to resolve DRPs. In this context, these activities are 
Please answer the questions in the suggested form

a) closed question - if applicable, use number $\mathbf{1}$
b) open questions - please provide the answers citing relevant data and information

1. Type and size of the pharmacy:

1.1 Type

a) community pharmacy which is part of an association, alliance or cooperative

b) community pharmacy which is part of a pharmacy network (one brand)

c) community pharmacy which is not part of an association, alliance or cooperative or a network

e) non-state hospital pharmacy

d) state-owned hospital pharmacy

1.2 Number of professional employees (pharmacists and lab workers) employed on part- and full-time basis:

a) $1-5$

b) $6-1$

c) $>10$

2. Municipality/town/region in which the pharmacy is located:

a) municipality/town

3. Number of years of professional experience (1 person per pharmacy):

b) $10-19$

) $10-19$

c) $20-29$

4. Which professional/scientific area(s) are you specialized in? Please specify:

a) I am specialized in:

5. Is your pharmacy equipped with an appropriate space for consultations and therapy assessment, or specialized workplace where infort 92/2012 Coll. \& SK Decree No 129/2012 Coll.)

5.1 Consultation space or room

a) yes
b) no

5.2 Discrete zone

a) yes

b) no
5.3 How often do you provide consultations to patients beyond the usual drug dispension-related activities?

a) daily $1-3$ times per week

c) $1-3$ times per month

6. Please provide information on your 2 or 3 most frequently used sources of information related to medicines

6. Please provide information on your 2 or 3 most frequently used sources of information related to medicines
and recommended procedures of the Czech Pharmacists' Association and how frequently you use them: and recommended procedures of the Czech Pharmacists' A

Recommended procedures

7. Do you cooperate with a medical or healthcare practitioner in your pharmacy regarding DRPs?

a) yes
b) no

Note: DRPs means Drug Related Problems (e.g. interactions, incorrect use, attitude and behavior of patients, etc.)

7.1 If yes, how often do you do so?

a) daily

b) 2-3 times per week

d) $5-2$ times per month

8. Do you take records of interventions in your pharmacy (DRPs)?

a) yes
b) no

8.1 If yes, in which form do you take such records?

electronic forn

b) paper form

9. Do you have a system in your pharmacy that counts the number of patients and their revisits?

a) yes

9.1 If yes, please make an estimate of proportions of loyal vs random patients in your pharmacy:

a) loyal patients ( $(\mathrm{\%})$
b) random patients $(\mathrm{v} \%)$

\section{Figure 1. Questionnaire to community pharmacists.}

understood as going beyond the scope of primary (necessary) information provided by individual pharmacists about safe and appropriate use of dispensed medicines.

V. Offering support by pharmacists to follow a recommended pharmacotherapy in order to achieve:

a. Compliance, adherence, persistence and concordance. The terms and definitions refer to a literature review by Vrijens et al. (2012);

b. Reducing the risk of non-adherence - we used four item Morisky Medication Adherence Scale (Morisky et al., 1986), which we modified from the pharmacist's perspective, who responds according to his/her experience and common practice in communication with patients on medication use (Fig. 1, question 12/a-d).
10. In which manner do you offer support/motivate patients towards higher compliance in the recommended herapy? (multiple choice possible)

(a)

b) suggesting a cheaper alternative in the alternative form of generic substitution

al counselling)

d) support programs for a larger group of patients - e.g. group discussion, audio-visual program

e) other (please specify):

Note: Compliance means that the patient's behavior follows the treatment recommendation by a healthcar professional (medicine practitioner, nurse, etc.)

11. Do you provide any consultations to patients, or individual counselling on use of medicine?

a) yes

11.1 If yes, how often do you do so?

a) daily

b) daily $2-3$ times per week

b) $2-3$ times per week
c) $1-2$ times per month
d) $5-6$ times per month

12. How do you usually identify the risk of non-adherence? What questions do you usually ask the patient? The questions related to: (multiple choice is possible)

a) forgetfulness

b) missing dosage of medicine in case of adverse or side effects occur

c) early termination of therapy

13. In what manner do you usually offer support for adherence? (multiple choice is possible)

a) we provide information about use of medicine

b) we provide the information related to the use of medicine and verify if the patient understands the information c) we recommend to patient his/her appropriate system to monitoring and recording use of medicines d) other (please specify)

e) we do not offer such support
Note: Adherence means an affirmative attitude on behalf of patient towards treatment, acting in accordance with the pharmacotherapy recommended by the healthcare practitioner. It significantly influences the therapeutic value of the prescribed medicin

14. In what manner do you offer support for persistence - continuity of treatment - throughout the recommended period of therapy? (multiple choice is possible)

we engage the patient by asking questions related to his or her readiness to undertake a long-term treatment

b) we agree with the patient on a continuous cooperation

we have suspect that the patient deliberately adjusts the length of the recommended pharmacotherapy

d) other (please specify)

e) we do not offer such support
Note: Persistence - means continuity throughout the entire treatment (i.e. patient uses medicine throughout the entire therapy).

15. How do you increase trust in patients in order to achieve their concordance? (multiple choice is possible) a) we provide personal counselling related to the selected drug form, application and dosage

b) we consult with patient the recommended medication dose titration (e.g. due to skin irritation)

c) we identify with the patient his or her options for a routine use of medicines in accordance with the maintenance therapy recommended by a medicine practitioner (e.g. system of notifications, personal monitoring

d/ we engage the patient in different way (please specify):

e) we do not deal with such issue

Note: Concordance means a partnership between patient and pharmacist that leads to an agreement on why and how to use the relevant medicines. Unlike compliance, concordance represents an agreement based on a patien's own conviction, reached as a restlt of an indidual approach and it is based on the provision of adequate and other relevant factors.

\section{Statistical methodology}

To process the results of the questionnaires, we used methods of descriptive and inductive statistics. The core of the questionnaire was formed around the questions of a dichotomic or polytomic character. The final quantities were summarized in a contingency table. To test associations, we used the chi-squared test $\left(\mathrm{X}^{2}\right)$ or exact tests (Fisher and FisherFreeman-Halton tests). The differences between groups were tested by the t-test and, in relevant cases, by non-parametric methods (Mann-Whitney test for analysis of variations). We analysed the degree of associations with correlation coefficients: Cramér's V and Goodman and Kruskal's gamma. For certain comparisons, we introduced an odds ratio (OR) with the respective confidence interval, $95 \%(95 \% \mathrm{CI})$. In all 
Table 1. Consultancy and resolving DRPs by community pharmacists.

\begin{tabular}{|c|c|c|c|c|c|c|c|c|c|c|}
\hline $\begin{array}{l}\text { Consul- } \\
\text { tations } \\
\text { on DRPs }\end{array}$ & \multicolumn{2}{|c|}{ DRPs } & \multirow{2}{*}{$\begin{array}{c}\text { CZ score } \\
\text { SUM }\end{array}$} & & \multirow[t]{2}{*}{$\begin{array}{c}\text { Cohen } \\
\text { Kappa } \\
(95 \% \text { CI) }\end{array}$} & \multicolumn{2}{|c|}{ DRPs } & \multirow{2}{*}{$\begin{array}{c}\text { SK score } \\
\text { SUM }\end{array}$} & P & $\begin{array}{c}\text { Cohen } \\
\text { Kappa } \\
(95 \% \mathrm{Cl})\end{array}$ \\
\hline & $\mathrm{NO}$ & YES & & \multirow{4}{*}{0.796} & & $\mathrm{NO}$ & YES & & \multirow{4}{*}{0.00004} & \multirow{4}{*}{$\begin{array}{c}0.554 \\
(0.183 \text { to } \\
0.926)\end{array}$} \\
\hline Yes & 19 & 62 & 81 & & \multirow{3}{*}{$\begin{array}{c}-0.074 \\
(-0.241 \text { to } \\
0.092)\end{array}$} & 4 & 34 & 38 & & \\
\hline No & 6 & 30 & 36 & & & 3 & 0 & 3 & & \\
\hline Total & 25 & 92 & 117 & & & 7 & 34 & 41 & & \\
\hline
\end{tabular}

cases, we tested at a significance level of alpha $\leq 0.05$. We performed the analyses with StatsDirect ${ }^{\bigotimes} 2.8 .0$ (StatsDirect Ltd., Cheshire, UK) and Microsoft Office Excel 2010 (Microsoft Corporation).

\section{Study's limitations}

The legal framework for personal data protection of patients, their unavailable medication history and non-existence of the e-Health in CZ and SK limited our study with regards to community pharmacist's support on specific patient, his/ her specific type of problem/s and pharmacotherapeutic outcome/s. Therefore, collected data from the pharmacists' responses give an estimate on the medication adherence promotion provided in respective pharmacies participating in the questionnaire survey.

\section{RESULTS}

\section{Consultations, counselling and DRPs}

The majority of pharmacists in our data set stated that they provide consultations or counselling to patients (CZ: 69.2\%, SK: $92.7 \%)$. We investigated whether these activities could be associated with resolving DRPs. Unlike CZ, in SK there was a significant and high concurrence in answers (answer type yesyes) that showed that if SK pharmacists provide consultations, they do so in order to help patients in finding solution to DRPs and, on the other hand, those pharmacists that do not provide consultations, do not engage with DRP-solving activities (answer type no-no), $\mathrm{P}<0.0001$ (Table 1).

In registering DRPs, we found a statistically significant difference in favour of SK pharmacists $(P=0.008)$. Pharmacists do register DRPs, although there is no e-Health currently at place in either country. Such registrations therefore tend to be non-systematic and rare. Due to the small number of responses, we could not assess to what extent DRPs are registered in either paper or electronic form. In both countries, pharmacists deal with DRPs at a similar rate of frequency, usually either daily or 2 to 3 times per week $(P=0.052)$.

Just about half of the pharmacists (50.8\%) in both countries register loyal patients and their revisits. Loyal patients represented more than $50 \%$ of all patients visiting pharmacies.
We did not find a correlation between revisits of patients and resolving DRPs in the data sets.

\section{Support of medication adherence}

Each evaluated activity of pharmacists aimed at an improvement of medication adherence, persistence or concordance, was scored with one point. The overall relative score for all activities combined could range from 0 to 4 points. The more intensive the support of a pharmacist was, the higher the score. We did not observe significant differences between CZ and SK in support of the adherence, persistence or concordance. Support for adherence reached the average level with the highest scoring value in both countries. Support for adherence (CZ: 1.95; SK: 1.93) was significantly higher than support for persistence (CZ: 1.18;SK: 1.07), and was also higher than support for concordance (CZ: 1.28;SK: 1.27), (Fig. 2). We used the adopted Morisky scale to estimate with what intensity non-adherence risk could be reduced. We evaluated this from the perspective of the pharmacist, who addresses one or more critical areas of a patient's attitude and behaviour, which can help to uncover patient's compliance with a prescribed pharmacotherapy. In CZ, we measured that the overall average score was marginally higher than for SK. It reached the medium degree of support in both data sets [CZ: 2.29 and SK: 2.22; $\mathrm{P}=0.739 ; 95 \% \mathrm{Cl}$ for the difference in mean scores went from $(-0.35)$ to 0.49$]$. The difference was attributed to a higher score achieved by $\mathrm{CZ}$ pharmacists concerning the reduction of any risk that patient would not comply with the recommended time of medication use $(P=0.032$; the lower and upper limits of the $95 \% \mathrm{Cl}$ for the odds ratio were 1.02 and 5.09). Other risk factors were comparable with SK. These include forgetfulness, missing medication or termination of medication use due to adverse reaction or, on the contrary, because therapeutic effect has already been attained (Table 2 ).

Relationship analysis between estimated support for medication adherence and technical and personnel characteristics of pharmacies

The survey demonstrated that geographic parameters of location (in relation to the number of inhabitants) or size of pharmacies by the number of professional employees, namely, 
Table 2. Non-adherence risk reduction from pharmacists-responders' perspective in the most frequent areas.

\begin{tabular}{|c|c|c|c|c|c|}
\hline $\begin{array}{l}\text { Reduction of risk } \\
\text { of non-adherence }\end{array}$ & $\mathbf{C Z}$ & SK & TOTAL & $\mathbf{p}$ & $\begin{array}{c}\text { OR } \\
95 \% \mathrm{Cl}\end{array}$ \\
\hline \multicolumn{6}{|c|}{ 1. Forgetfulness } \\
\hline Yes & 64 & 24 & 88 & \multirow{2}{*}{0.717} & \multirow{2}{*}{$\begin{array}{c}0.855 \\
0.387 \text { to } 1.861\end{array}$} \\
\hline No & 53 & 17 & 70 & & \\
\hline \multicolumn{6}{|c|}{ 2. Not complying with the recommended timing of usage of medicines } \\
\hline Yes & 85 & 22 & 107 & \multirow{2}{*}{0.032} & \multirow{2}{*}{$\begin{array}{c}2.294 \\
1.022 \text { to } 5.091\end{array}$} \\
\hline No & 32 & 19 & 51 & & \\
\hline \multicolumn{6}{|c|}{ 3. Missing dosages of medicines in case of occurrence of adverse or side effects } \\
\hline Yes & 67 & 27 & 94 & \multirow{2}{*}{0.361} & \multirow{2}{*}{$\begin{array}{c}0.694 \\
0.304 \text { to } 1.541\end{array}$} \\
\hline No & 50 & 14 & 64 & & \\
\hline \multicolumn{6}{|c|}{ 4. Early termination of treatment after achieving the desired therapeutic effect } \\
\hline Yes & 52 & 18 & 70 & \multirow{2}{*}{$>0.999$} & \multirow{2}{*}{$\begin{array}{c}1.022 \\
0.470 \text { to } 2.241\end{array}$} \\
\hline No & 65 & 23 & 88 & & \\
\hline Total score value & 2.29 & 2.22 & & 0.739 & \multirow{2}{*}{$\begin{array}{c}95 \% \mathrm{Cl} \\
-0.350 \text { to } 0.492\end{array}$} \\
\hline $\mathrm{n}$ & 117 & 41 & 158 & & \\
\hline
\end{tabular}

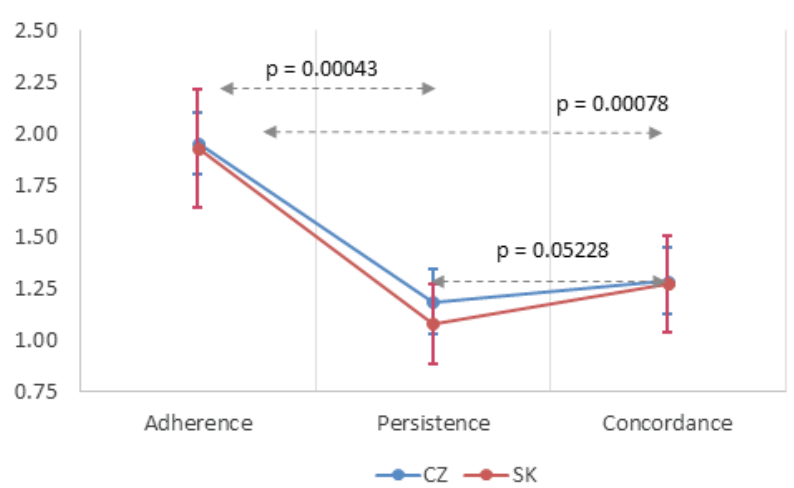

Figure 2. Support of patients' attitude to follow recommended pharmacotherapy.

did not correlate with the estimated degree of support for medication adherence.

The CZ and SK sets of pharmacies are equipped with technical equipment, that is, a discrete zone given by law and eventually, a consultation room-dedicated for consultancy purpose by law in $C Z$, not regulated in SK. The SK pharmacists, while using consultation rooms, were engaged more with adherencerelated issues as compared to $C Z(P=0.037$; Cramér's $V=0.37)$. On the other hand, the $\mathrm{CZ}$ pharmacists use the consultancy rooms - if they are equipped with - more frequently than SK pharmacists, although at the margin of significance $(P=0.058)$.

Majority of the survey's respondents had a qualification in pharmacy (CZ: $88 \%$; SK: $83 \%$ ). As far as the number of years of professional experience (practice) of the pharmacists (i.e., the respondents) is concerned, our data showed that the respondents mostly had between 20 and 29 years of practice in both data sets. When it comes to the number of years of professional experience, we found a difference between
SK and CZ - there is a stronger relationship between the number of years of experience and adherence support in SK $(p=0.029)$, whereas we could not establish a correlation in $C Z(P=0.175)$. Moreover, in the SK data set, we also found a strong linear relationship between the number of years of professional experience and medication adherence support $(P=0.0496)$ (Table 3$)$. We also found a strong correlation between the preferential source of information for pharmacists as the Recommended Procedures authorized by the Czech Chamber of Pharmacists (2010) and support for adherence by CZ pharmacists $(P=0.005)$, in comparison to SK pharmacists, where a similar source of information was not available. We observed, that majority of pharmacists cooperated with healthcare practitioners in solving the DRPs in both countries (CZ: 78.6\%; SK: $82.9 \%$; $\mathrm{P}=0.51 ; 95 \% \mathrm{Cl} 0.253$ to 2.023 ) (Table 4). A close relationship between adherence and DRPs was confirmed in the $C Z$ data set $(P=0.00015)$ as compared to the SK data set, despite Cramer's V showing a weak association (0.334). It is possible, that if data sets were larger, we would not observe the difference between $C Z$ and SK data sets. (Table 5).

\section{DISCUSSION}

Results of the questionnaire survey matched with our prediction that pharmacists had been involved in the medication adherence support, as similarly published by other authors (Lau et al., 2010, Santschi et al., 2012, Vlček, et al., 2009). We assume that pharmacists can support patient adherence and they are able to reduce the risk of patients' non-adherence, reaching in average the medium degree. We found that support for medication adherence has been provided to a greater degree than support for other forms of patients' adherence attitudes towards treatment, such as persistence and concordance. (Herein, we refer to 
Support of medication adherence by community pharmacists in Czech and Slovak Republics ...

Table 3. Association between the number of years of pharmacists' practice and support for medication adherence.

\begin{tabular}{|c|c|c|c|c|c|c|c|c|c|}
\hline & \multicolumn{9}{|c|}{$\begin{array}{c}\text { Support to medication adherence } \\
\text { ( } n=\text { number of pharmacists-responders) }\end{array}$} \\
\hline $\begin{array}{c}\text { Number } \\
\text { of } \\
\text { practice } \\
\text { (years) }\end{array}$ & $\begin{array}{l}\text { We inform } \\
\text { patient } \\
\text { about } \\
\text { use of } \\
\text { medicines } \\
\text { (n) }\end{array}$ & $\begin{array}{l}\text { We provide } \\
\text { information } \\
\text { and verify } \\
\text { if patient } \\
\text { understands } \\
\text { it }(\mathrm{n})\end{array}$ & $\begin{array}{l}\text { We recommend } \\
\text { that patient } \\
\text { creates his or her } \\
\text { own system of } \\
\text { monitoring of use } \\
\text { of medicines (n) }\end{array}$ & $\begin{array}{l}\text { Other } \\
\text { (n) }\end{array}$ & $\begin{array}{l}\text { We do not } \\
\text { provide } \\
\text { support to } \\
\text { adherence } \\
\text { (n) }\end{array}$ & $\underset{(n)}{\operatorname{Sum}}$ & $\begin{array}{l}\text { Fisher- } \\
\text { Freeman- } \\
\text { Halton } \\
\text { exact } \\
\text { P }\end{array}$ & $\begin{array}{c}\text { Good } \\
\text { man- } \\
\text { Kruskal } \\
\text { gamma }\end{array}$ & $P$ \\
\hline \multicolumn{10}{|l|}{ CZ } \\
\hline $0-9$ & 1 & 3 & 7 & 3 & 4 & 18 & \multirow{5}{*}{0.294} & \multirow{5}{*}{$\begin{array}{c}0.135 \\
(0.06 \text { to } \\
0.33)\end{array}$} & \multirow{5}{*}{0.175} \\
\hline $10-19$ & 5 & 5 & 17 & 9 & 4 & 40 & & & \\
\hline $20-29$ & 2 & 7 & 8 & 11 & 7 & 35 & & & \\
\hline$>29$ & 2 & 0 & 9 & 10 & 3 & 24 & & & \\
\hline Total & 10 & 15 & 41 & 33 & 18 & 117 & & & \\
\hline \multicolumn{10}{|l|}{ SK } \\
\hline $0-9$ & 1 & 4 & 0 & 2 & 0 & 7 & \multirow{5}{*}{0.024} & \multirow{5}{*}{$\begin{array}{c}0.348 \\
(0.04 \text { to } \\
0.66)\end{array}$} & \multirow{5}{*}{0.029} \\
\hline $10-19$ & 1 & 1 & 8 & 2 & 1 & 13 & & & \\
\hline $20-29$ & 1 & 1 & 0 & 3 & 2 & 7 & & & \\
\hline$>29$ & 1 & 3 & 2 & 3 & 5 & 14 & & & \\
\hline Total & 4 & 9 & 10 & 10 & 8 & 41 & & & \\
\hline
\end{tabular}

Table 4. Interdisciplinary teamwork between community pharmacists and healthcare practitioners.

\begin{tabular}{|c|c|c|c|c|c|c|}
\hline \multicolumn{7}{|c|}{$\begin{array}{l}\text { Cooperation between pharmacist and a healthcare practitioner on DRPs } \\
\text { ( } n=\text { number of pharmacists-responders })\end{array}$} \\
\hline \multirow{2}{*}{ Cooperation } & \multicolumn{2}{|c|}{$\mathrm{CZ}$} & \multicolumn{2}{|c|}{ SK } & \multirow[b]{2}{*}{ Total } & \multirow[b]{2}{*}{ Fisher exact } \\
\hline & $\mathrm{n}$ & $\%$ & $\mathrm{n}$ & $\%$ & & \\
\hline Yes & 92 & 78.6 & 34 & 82.9 & 126 & \multirow{3}{*}{$\begin{array}{c}\mathrm{P}=0.51 \\
95 \% \mathrm{Cl}: \\
0.253 \text { to } 2.023\end{array}$} \\
\hline No & 25 & 21.4 & 7 & 17.1 & 32 & \\
\hline Total & 117 & 100.0 & 41 & 100.0 & 158 & \\
\hline
\end{tabular}

Table 5. Adherence support and resolving DRPs by community pharmacists.

\begin{tabular}{|c|c|c|c|c|c|c|c|c|}
\hline & \multicolumn{8}{|c|}{$\begin{array}{l}\text { Association between adherence support and DRPs resolving by pharmacists } \\
\text { ( } \mathrm{n}=\text { number of pharmacists-responders })\end{array}$} \\
\hline $\begin{array}{c}\text { Adherence } \\
\text { and } \\
\text { DRPs }\end{array}$ & $\begin{array}{l}\text { We do not } \\
\text { provide } \\
\text { support to } \\
\text { adherence } \\
\text { We inform } \\
\text { patient } \\
\text { about } \\
\text { use of } \\
\text { medicines } \\
\text { (n) } \\
\end{array}$ & $\begin{array}{l}\text { Forgetfulness } \\
\text { (n) }\end{array}$ & $\begin{array}{l}\text { Not complying } \\
\text { with the } \\
\text { recommended } \\
\text { timing of } \\
\text { usage of } \\
\text { medicines }(n)\end{array}$ & $\begin{array}{c}\text { Missing } \\
\text { dosages of } \\
\text { medicines } \\
\text { in case of } \\
\text { occurrence } \\
\text { of adverse or } \\
\text { side effects (n) }\end{array}$ & $\begin{array}{c}\text { Early } \\
\text { termination } \\
\text { of treatment } \\
\text { after achieving } \\
\text { the desired } \\
\text { therapeutic } \\
\text { effect }(n)\end{array}$ & $\underset{(n)}{\operatorname{Sum}}$ & \begin{tabular}{|} 
Fisher- \\
Freeman- \\
Halton \\
exact \\
P
\end{tabular} & Cramér's \\
\hline \multicolumn{9}{|l|}{ CZ } \\
\hline Yes & 3 & 14 & 28 & 30 & 17 & 92 & \multirow{3}{*}{0.00015} & \multirow{3}{*}{0.4553} \\
\hline No & 7 & 1 & 13 & 3 & 1 & 25 & & \\
\hline Total & 10 & 15 & 41 & 33 & 18 & 117 & & \\
\hline \multicolumn{9}{|l|}{ SK } \\
\hline Yes & 4 & 7 & 7 & 10 & 6 & 34 & \multirow{3}{*}{0.3561} & \multirow{3}{*}{0.3345} \\
\hline No & 0 & 2 & 3 & 0 & 2 & 7 & & \\
\hline Total & 4 & 9 & 10 & 10 & 8 & 41 & & \\
\hline
\end{tabular}


terms and definitions on medication adherence used in the questionnaire by Vrijens et al. (2012)). As the issues of persistence and concordance are more complicated - the processes are more complex and demanding (professionally, in terms of time or work organization). We estimate that pharmacists supported patients only at a low degree of intensity in these activities. In general, this degree of support could change if pharmacists were more actively, or even pro-actively, engaged in the cooperation with patients and healthcare practitioners in both countries. It is most probably associated with education and further training, their motivation, as well as availability of specific intervention package/s motivational interviews with patients, patients' education, home medicines review, dose administration aid, medication use review, etc.), enabling the pharmacists to improve patient adherence (Lau et al., 2010, Salvo \& CannonBreland, 2015).

We found that the location of a pharmacy and the number of inhabitants in a location does not influence support for medication adherence in either country (for pharmacies located in larger cities or smaller municipalities). As far as technical factors were concerned, we focused on discrete zones and consultation rooms and their use. In SK, where discrete zones are mandatory components of pharmacies (Decree No 129/2012), consultation rooms are additional spaces not required by law. The outcomes suggested that there is an association between support for adherence (DRPs) and the usage of consultation rooms, which has been stated by Masaryková et al., 2015 too. In CZ, only pharmacies that actively provide consultations and counselling have such consulting rooms (Decree No 92/2012). Despite strong differences in terms of the legislative requirements in the two countries, our finding showed that CZ pharmacies use consultation rooms more frequently - though the frequency was only marginally higher than SK pharmacies. This finding indicates that legislation is not the unique factor that influences the extent to which pharmaceutical care is provided. In this respect, other factors, both personal and professional, also have significant importance. The difference in the legislative and societal development of pharmaceutical care in the two countries reveals potential that we could elaborate upon to increase support for medication adherence by pharmacists.

From the perspective of personnel characteristics of pharmacy, the degree of support for adherence was not dependent upon the number of professional workers. However, in the SK data set, we found that the number of years of professional experience increase the probability that the pharmacists approach patients, discuss a prescribed treatment and try to help to resolve patients'DRPs. We assume, that SK pharmacies, in comparison to $C Z$ pharmacies, provide such a kind of consultations that are closely linked to resolving DRPs in collaboration with healthcare practitioners. The importance of interdisciplinary cooperation in DRPs (most notably effective interaction between community pharmacist and healthcare practitioner/clinic setting) has been often highlighted at scientific symposia and discussed by more authors (Pape et al., 2011, Rojaz-Fernandez et al., 2014). According to recently published data from SK pharmacies by Masaryková et al., the DRPs most frequently relate to poor treatment efficacy and therapy costs (2015). Non-adherence very likely represents the common denominator of these problems. The risk of non-adherence should be detected and resolved responsibly, whereas cooperation between pharmacists and healthcare practitioners gives a solid ground for furnishing effective and prompt solutions to the identified problems. To examine other possibilities of how to increase support for patient adherence, the specific profile of risk patients could be considered, which the pharmacists could focus on.

Our findings indicated that there is a close association between support for medication adherence and personnel capacities (factors) of pharmacy. These factors mostly concern the professional experience of pharmacists and the quality of consultation and counselling, which might be efficiently supported by training and/or authorized sources of recommendations for pharmacists such as Recommended Procedures of the Czech Pharmacists' Association available since 2010. Recently, the Association opened new courses in DRPs, enabling additional services, which include recommendations in terms of dosage and therapeutic regime. The Association is planning to extend its recommendations into other areas of pharmaceutical professional consultancy. Since 2013, the Czech Chamber of Pharmacists offers a mobile application mapping the pharmacies with consultation rooms. All these initiatives, as mentioned hereby, represent useful electronic tools that could enable the pharmacists to address patients' adherence and vice-versa, in more effective way.

In general, we can assume that the support for medication adherence is provided in both CZ and SK pharmacies. It reaches a medium degree and develops in the right direction - towards concordance in the pharmacist-patient relationship. The responses to our survey suggest that the development of interdisciplinary cooperation with other healthcare professionals, mostly medical practitioners, remains challenging (non-systematic and rare registration of DRPs). The e-Health system will soon be introduced in both countries, and is expected to become an important factor in this dimension, as it will enable connection with relevant data and information. It is also recognizable that recommended practice, education in DRPs and life-long education of pharmacists represent important pre-requisites for further development of pharmaceutical care in medication adherence support.

\section{CONCLUSION}

We analysed the current state of provision of counselling and consultation services aimed at supporting medication adherence by community pharmacies through a questionnaire 
survey in CZ and SK. Based on the pharmacists' responses, we assumed a medium degree of support for patient adherence, predominantly provided in order to resolve DRPs in both countries. We can also point out that education and experience for pharmacists could be one of the crucial factors in ensuring support for adherence. Its progress might also be accelerated through the use of information technologies, as research in multiple countries has also demonstrated recently. We conclude that our findings can offer a solid base for further investigations aimed at evidence-based interventions in this area, and so to contribute to pharmaceutical care development.

\section{DECLARATIONS}

\section{List of Abbreviations}

CI: Confidence Interval CZ: Czech or Czech Republic DRPs: Drug-related problems e-Health: Electronic and computerized healthcare system FaF UK: Faculty of Pharmacy, Comenius University in Bratislava or Faculty of Pharmacy in Hradec Králové, Charles University OR: Odds Ratio SK: Slovak or Slovak Republic WHO: World Health Organization $\mathbf{X}^{2}$ : chi-squared test

\section{ACKNOWLEDGEMENTS}

We thank the Academia, the Faculty of Pharmacy in Hradec Králové, Charles University and the Faculty of Pharmacy, Comenius University in Bratislava for their support to address and engaged community pharmacies into the study in both the Czech and Slovak Republics. We would also like to thank all community pharmacies taking part in the Survey.

\section{References}

[1] Czech Chamber of Pharmacists: Counselling in Pharmacies. (https://www.lekarnici.cz/Pro-verejnost/PORADENSTVI--KONZULTACE.aspx); (in Czech). Revised September 7, 2010. Accessed March 2016.

[2] Czech Chamber of Pharmacists: Pharmacies in Smartphones. (http://www.lekarnici.cz/Pro-verejnost/Informace-proverejnost/Lekarny-v-mobilu.aspx); (in Czech). Revised November 15, 2013. Accessed March 2016.

[3] Decree No 92/2012 Coll. on the requirements for the minimum technical and material equipment of health facilities and home care contact centers. Ministry of Health. (https://portal.gov.cz/ app/zakony/zakonPar.jsp?idBiblio=77185\&nr=92 2F2012\&rpp= 15\#local-content); (in Czech). Revised March 15, 2012. Effective April 1, 2012. Accessed March 4, 2016.

[4] Decree No 129/2012 Coll. on the requirements for good pharmacy practice. Ministry of Health of the Slovak Republic. (http://jaspi. justice.gov.sk/jaspiw1/htm zak/jaspiw mini zak zobraz skup1. asp?skupina=1); (in Slovak).Revised April 12, 2012. Effective May 15, 2012. Accessed March 4, 2016.

[5] FIP. International Pharmaceutical Federation:FIP/WHO Joint Guidelines on Good Pharmacy Practice - Standards for Quality Services. 2011. (http://www.fip.org/www/uploads/database_file. php?id=331\&table id=). Accessed March 4, 2016.

[6] Kriška M, GajdošíkJ, Dukát A, Bernadič M. Zlyhanie farmakoterapie - možnosti prevencie. Bratislava: Slovak Academic Press; 2015; (in Slovak).

[7] Lau R, Stewart K, McNamara KP et al. Evaluation of a community pharmacy-based intervention for improving patient adherence to antihypertensives: a randomised controlled trial. BMC Health Services Research. 2010; doi: 10.1186/1472-6963-10-34. PubMed

[8] Leporini C, De Sarro G, Russo E. Adherence to therapy and adverse drug reactions: is there a link? Expert Opinion on Drug Safety. 2014; doi: 10.1517/14740338.2014.947260. PubMed

[9] Masaryková L, Fulmeková M, Lehocká L', Fazekaš T. Identifying and solving drug-related problems in terms of the community pharmacists. Čes slov farm. 2014;63:32-39; (in Slovak).

[10] Morisky DE, Green LW, Levine DM. Concurrent and predictive validity of a self-reported measure of medication adherence. Med Care. 1986;24(1):67-74.

[11] Pape GA, Hunt JS, Butler KL et al. Team-based care approach to cholesterol management in diabetes mellitus: two-year cluster randomized controlled trial. Arch Intern Med.2011;171(16):1480-6. PubMed

[12] PCNE - Pharmaceutical Care Network Europe Foundation: The PCNE Classification V5.01. 2006. (http://www.pcne.org). Published 2006. Accessed March 4, 2016.

[13] Rojas-Fernandez CH, Patel T, Lee L. An Interdisciplinary Memory Clinic: A Novel Practice Setting for Pharmacists in Primary Care. Ann Pharmacother. 2014 Jun;48(6):785-95. PubMed

[14] Salvo MC, Cannon-Breland ML. Motivation interviewing for medication adherence. J Am Pharm Assoc. 2015 JulAug;55(4):e354-61. PubMed

[15] Santschi V, Chiolero A, Paradis G, Colosimo AL, Burnand B. Pharmacist interventions to improve cardiovascular disease risk factors in diabetes: a systematic review and meta-analysis of randomized controlled trials. Diabetes Care. 2012;35(12):2706-17. Pub Med 
[16] Spinewine A, Fialová D, Byrne S. The Role of the Pharmacist in Optimizing Pharmacotherapy in Older People. Drugs Aging. 2012;29(6):495-510. PubMed

[17] Středa L, Hána K. (eHealth and telemedicine). Praha: Grada Publishing; 2016; (in Czech).

[18] Sun AP, Kirby B, Black C, Helms PJ, Bennie M, McLay JS. Unplanned medication discontinuation as a potential pharmacovigilance signal: a nested young person cohort study. BMC Pharmacology and Toxicology. 2014; doi:10.1186/2050-6511-15-11. PubMed

[19] Vlček J, Malý J, Dosedel M. (Pharmaceutical care of patients with diabetes mellitus and its relationship to clinical pharmacy). Vnitr Lek. 2009;Apr; 55(4):384-8; (in Czech). PubMed

[20] World Health Organization (WHO): Chronic diseases and health promotion: Adherence to long-term therapies: evidence for action. (http://www.who.int.). Published 2003. Accessed March 4, 2016.

[21] Vrijens B, De Geest S, Hughes DA et al. A new taxonomy for describing and defining adherence to medications. $\mathrm{Br} J$ Clin Pharmacol. 2012 May;73(5):691-705. PubMed

[22] Taitel M, Jiang J, Rudkin K, Ewing S, Duncan I. The impact of pharmacist face-to-face counseling to improve medication adherence among patients initiating statin therapy. Patient Preference and Adherence. 2012;6:323-329. PubMed 